\title{
Tensor-Based Methods for Blind Spatial Signature Estimation in Multidimensional Sensor Arrays
}

\author{
Paulo R. B. Gomes, ${ }^{1}$ André L. F. de Almeida, ${ }^{1}$ João Paulo C. L. da Costa, ${ }^{2,3,4}$ \\ João C. M. Mota, ${ }^{1}$ Daniel Valle de Lima, ${ }^{2}$ and Giovanni Del Galdo ${ }^{3,4}$ \\ ${ }^{1}$ Department of Teleinformatics Engineering, Federal University of Ceará, Fortaleza, CE, Brazil \\ ${ }^{2}$ Department of Electrical Engineering, University of Brasília, DF, Brasília, Brazil \\ ${ }^{3}$ Institute for Information Technology, Ilmenau University of Technology, Ilmenau, Germany \\ ${ }^{4}$ Fraunhofer Institute for Integrated Circuits IIS, Erlangen, Germany \\ Correspondence should be addressed to Paulo R. B. Gomes; paulo@gtel.ufc.br
}

Received 10 September 2016; Accepted 15 January 2017; Published 15 February 2017

Academic Editor: Elias Aboutanios

\begin{abstract}
Copyright ( 2017 Paulo R. B. Gomes et al. This is an open access article distributed under the Creative Commons Attribution License, which permits unrestricted use, distribution, and reproduction in any medium, provided the original work is properly cited.

The estimation of spatial signatures and spatial frequencies is crucial for several practical applications such as radar, sonar, and wireless communications. In this paper, we propose two generalized iterative estimation algorithms to the case in which a multidimensional $(R-D)$ sensor array is used at the receiver. The first tensor-based algorithm is an $R$-D blind spatial signature estimator that operates in scenarios where the source's covariance matrix is nondiagonal and unknown. The second tensor-based algorithm is formulated for the case in which the sources are uncorrelated and exploits the dual-symmetry of the covariance tensor. Additionally, a new tensor-based formulation is proposed for an $L$-shaped array configuration. Simulation results show that our proposed schemes outperform the state-of-the-art matrix-based and tensor-based techniques.
\end{abstract}

\section{Introduction}

High resolution parameter estimation plays a fundamental role in array signal processing and has practical applications in radar, sonar, mobile communications, and seismology. In light of this, several techniques have been developed to increase the accuracy of the estimated parameters, from which we may cite the classical Multiple Signal Classification (MUSIC) [1] and Estimation of Signal Parameters via Rotational Invariance Technique (ESPRIT) [2]. However, their performance can be further improved by exploiting the multidimensional structure of the data by means of tensor modeling, which can include several signal dimensions such as space, time, frequency, and polarization. Tensor decompositions have been successfully employed in array signal processing for parameters estimation since they provide better identifiability conditions when compared to conventional matrix-based methods. Another advantage of tensor-based methods is the so-called "tensor gain" which manifests itself with more precise parameter estimates due to the good noise rejection capability of tensor-based signal processing, as shown in [3-6].

In regards to tensor-based methods for blind spatial signatures estimation, the Parallel Factor (PARAFAC) analysis decomposition [7] is widely applied due to its welldefined conditions for uniqueness [8]. As seen in [9], an iterative technique for PARAFAC decomposition such as Trilinear Alternating Least Squares (TALS) can be applied to estimate the directions of arrival of the sources. Closed-form solutions such as the Standard Tensor ESPRIT (STE) [10] and Closed-Form PARAFAC [11] are also appealing, since these exploit the multidimensional structure in a noniterative fashion. Recently in [12], an iterative algorithm was proposed in a manner similar to Independent Component Analysis (ICA) based on the Orthogonal Procrustes Problem (OPP) and Khatri-Rao factorization [13] for a PARAFAC decomposition with dual-symmetry. This solution exploits the dual-symmetry property of the data tensor and can be applied in covariance-based array signal processing techniques. The method proposed in [14] is based on the Tucker 
decomposition [15] of a fourth-order covariance tensor and was elaborated for arrays with arbitrary structures, where a priori knowledge about the geometry of the sensor array is not required. However, a limitation of the method in [14] is the necessity of transmitting the same sequence of symbols in different time blocks, which results in a loss of spectral efficiency. The proposed solution is an algorithm for a multidimensional $(R-D)$ sensor array in which the different dimensions of the array are exploited, thus dismissing the need to transmit a repeated sequence as in [14], as will be detailed later.

In this paper, two tensor-based approaches to the estimation of spatial signatures are presented. By using the signals received on a $R-D$ sensor array, covariance tensors are calculated and solutions for correlated and uncorrelated sources are presented, respectively. For the former scenario, in which the source's covariance structure is nondiagonal and unknown, the covariance tensor of the received data is formulated as a Tucker decomposition of order $2 R$. Such a formulation yields a generalized Tucker model based $R$ $\mathrm{D}$ sensor array processing that deals with arbitrary source covariance structures. By assuming uncorrelated sources, we then show that the problem boils down to a PARAFAC decomposition, from which a method that exploits the dualsymmetry property of the covariance tensor is derived by considering the ideas rooted in [12]. For both Tucker and PARAFAC based models, the blind estimation of the spatial signatures is achieved by means of an alternating least squares (ALS) algorithm. The contributions of this paper are twofold: (i) we propose a covariance-based generalization of the Tucker decomposition for the blind spatial signature estimation problem with $R-D$ sensor arrays and (ii) we establish a link between dual-symmetry decompositions and techniques based on covariance-based array signal processing for parameter estimation. The performance of the proposed algorithms is evaluated by Monte Carlo simulations, corroborating their gains over competing state-of-the-art matrix-based and tensor-based techniques.

The rest of this paper is organized as follows: Section 2 briefly introduces tensor operations and decompositions. The signal model for an $R-D$ sensor array is then presented in Section 3. In Section 4 a novel covariance-based tensor model for the received data is formulated and our blind spatial signature estimation algorithms are formulated. In Section 5 an approach for $L$-shaped sensor arrays is proposed. The computational complexity of the proposed methods is analyzed in Section 6. In Section 7, the advantages and disadvantages of the proposed methods are discussed. Simulation results are provided in Section 8, and the conclusions are drawn in Section 9.

Notation. Scalar values are represented by lowercase letters $a$, vectors by bold lowercase letters a, matrices by bold uppercase letters $\mathbf{A}$, and tensors by calligraphic letters $\mathscr{A}$. The symbols $T, H, \dagger$, and $*$ represent the transpose, conjugate transpose, pseudoinverse, and complex conjugate operations, respectively. $\operatorname{diag}(\mathbf{a})$ operator generates a diagonal matrix from a vector a. The $i$ th row of $\mathbf{A} \in \mathbb{C}^{I \times R}$ is represented by $\mathbf{A}(i,:) \in \mathbb{C}^{1 \times R}$, while its $r$ th column is represented by
$\mathbf{A}(:, r) \in \mathbb{C}^{I \times 1} \cdot \operatorname{vec}(\mathbf{A})$ operator converts $\mathbf{A}$ into a vector $\mathbf{a} \in \mathbb{C}^{I R \times 1}$, while unvec ${ }_{I \times R}(\mathbf{a})$ converts a into a $I \times R$ matrix. $\mathbf{D}_{i}(\mathbf{A})$ stands for a diagonal matrix constructed from the $i$ th row of A. $\|\cdot\|_{F}$ stands for the Frobenius norm of a matrix or tensor. "o" operator stands for the vector outer product. The Kronecker product is represented by $\otimes$. The Khatri-Rao product between the matrices $\mathbf{A} \in \mathbb{C}^{I \times R}$ and $\mathbf{B} \in \mathbb{C}^{J \times R}$, represented by $\diamond$, is defined as

$$
\mathbf{A} \diamond \mathbf{B}=[\mathbf{A}(:, 1) \otimes \mathbf{B}(:, 1), \ldots, \mathbf{A}(:, R) \otimes \mathbf{B}(:, R)] .
$$

\section{Tensor Preliminaries}

In the following, we briefly introduce for convenience the basics on operations involving tensors and tensor decompositions, which refer to $[16,17]$. Firstly, we present the Tucker decomposition. Then, the PARAFAC decomposition is introduced and issues involving uniqueness are briefly discussed for both cases, which will be useful later. Then, we introduce the dual-symmetry property for these decompositions. The basic material presented in this section is exploited in later sections in the context of our blind spatial signature estimation problem.

2.1. Basic Tensor Operations. Let $\mathscr{X} \in \mathbb{C}^{I_{1} \times \cdots \times I_{N}}$ denote an $N$ th order tensor, $\left(i_{1}, \ldots, i_{N}\right)$ th entry of which is denoted by $x_{i_{1}, \ldots, i_{N}}$. The fibers are the higher-order analogues of matrix rows and columns. The $n$-mode fibers of $\mathscr{X}$ are vectors of size $I_{n}$ defined by fixing every index but $i_{n}$. The $n$-mode unfolding operation, denoted by $[\mathscr{X}]_{(n)}$, stands for the process of reordering the elements of $\mathscr{X}$ into a matrix by arranging its $n$-mode fibers to be the columns of the resulting matrix. The $n$-mode product between $\mathscr{X}$ and a matrix $\mathbf{A} \in \mathbb{C}^{J \times I_{n}}$ along of the $n$th mode, denoted by $\mathscr{X} \times{ }_{n} \mathbf{A}$, is a tensor of size $I_{1} \times \cdots \times I_{n-1} \times J \times I_{n+1} \times \cdots \times I_{N}$, obtained by taking the inner product between each $n$-mode fiber and the rows of the matrix A; that is, $[16,17]$

$$
\begin{aligned}
\mathscr{Y} & =\mathscr{X} \times{ }_{n} \mathrm{~A} \Longleftrightarrow \\
{[\mathcal{Y}]_{(n)} } & =\mathbf{A}[\mathscr{X}]_{(n)} .
\end{aligned}
$$

2.2. Tucker Decomposition. The Tucker decomposition [15] represents a tensor $\mathscr{X} \in \mathbb{C}^{I_{1} \times \cdots \times I_{N}}$ of order $N$ as a multilinear transformation of a core tensor $\mathscr{G} \in \mathbb{C}^{R_{1} \times \cdots \times R_{N}}$ by factor matrices $\mathbf{A}^{(n)}=\left[\mathbf{a}_{1}^{(n)}, \mathbf{a}_{2}^{(n)}, \ldots, \mathbf{a}_{R_{n}}^{(n)}\right] \in \mathbb{C}^{I_{n} \times R_{n}}$ along each mode $n=1,2, \ldots, N$. In scalar form, the $N$ th order Tucker decomposition is given by

$$
x_{i_{1}, i_{2}, \ldots, i_{N}}=\sum_{r_{1}=1}^{R_{1}} \sum_{r_{2}=1}^{R_{2}} \cdots \sum_{r_{N}=1}^{R_{N}} g_{r_{1}, r_{2}, \ldots, r_{N}} a_{i_{1}, r_{1}}^{(1)} a_{i_{2}, r_{2}}^{(2)} \cdots a_{i_{N}, r_{N}}^{(N)}
$$

where $a_{i_{n}, r_{n}}^{(n)}$ is $\left(i_{n}, r_{n}\right)$ th entry of the $n$th mode factor matrix $\mathbf{A}^{(n)} \in \mathbb{C}^{I_{n} \times R_{n}}$ and $g_{r_{1}, r_{2}, \ldots, r_{N}}$ is $\left(r_{1}, \ldots, r_{N}\right)$ th entry of the core tensor $\mathscr{G} \in \mathbb{C}^{R_{1} \times \cdots \times R_{N}}$. Using $n$-mode product notation, the Tucker decomposition can be written as

$$
\mathscr{X}=\mathscr{G} \times{ }_{1} \mathbf{A}^{(1)} \times_{2} \mathbf{A}^{(2)} \cdots \times{ }_{N} \mathbf{A}^{(N)},
$$


which admits the following factorization in terms of the factor matrices and core tensor:

$$
\begin{aligned}
& {[\mathscr{X}]_{(n)}} \\
& =\mathbf{A}^{(n)}[\mathscr{G}]_{(n)}\left(\mathbf{A}^{(N)} \otimes \cdots \mathbf{A}^{(n+1)} \otimes \mathbf{A}^{(n-1)} \otimes \cdots \mathbf{A}^{(1)}\right)^{T} .
\end{aligned}
$$

In general, the Tucker decomposition is not unique; that is, there are infinite solutions for $\mathbf{A}^{(n)}, n=1, \ldots, N$, and $\mathscr{G}$ that yield the same reconstructed version of the data tensor $\mathscr{X}$. However, in special cases where several elements of the core tensor are constrained to be equal to zero, that is, if the core tensor has some sparsity, the number of solutions may be finite, and the associated factor matrices and core tensor become unique up to trivial permutations and scaling ambiguities [18]. The Tucker based methods presented in Sections 4.2 and 5.1 belong to a special category where unique solutions exist.

2.3. PARAFAC Decomposition. The PARAFAC decomposition [7] expresses a tensor $\mathscr{X} \in \mathbb{C}^{I_{1} \times \cdots \times I_{N}}$ as a sum of $R$ rankone tensors; that is,

$$
\boldsymbol{X}=\sum_{r=1}^{R} \mathbf{a}_{r}^{(1)} \circ \mathbf{a}_{r}^{(2)} \circ \cdots \circ \mathbf{a}_{r}^{(N)},
$$

where $R$ is the number of factors, also known as the rank of the decomposition, and is defined as the minimum number of rank-one tensors that yield $\mathscr{X}$ exactly.

The Nth order PARAFAC decomposition (6) can be seen as a special case of the Tucker decomposition (4) with a core tensor $\mathscr{G}=\mathscr{I}_{N, R}$ and $R_{n}=R$ for $n=1, \ldots, N$. The elements of the $N$ th order identity tensor $\mathscr{I}_{N, R}$ are equal to one when all indices are equal and zero elsewhere. Using the $n$-mode product notation, the PARAFAC decomposition can be written as

$$
\mathscr{X}=\mathscr{I}_{N, R} \times{ }_{1} \mathbf{A}^{(1)} \times{ }_{2} \mathbf{A}^{(2)} \cdots \times{ }_{N} \mathbf{A}^{(N)},
$$

while the $n$-mode unfolding of $\mathscr{X}$ can be expressed as

$$
[\mathscr{X}]_{(n)}=\mathbf{A}^{(n)}\left(\mathbf{A}^{(N)} \diamond \cdots \mathbf{A}^{(n+1)} \diamond \mathbf{A}^{(n-1)} \diamond \cdots \mathbf{A}^{(1)}\right)^{T} .
$$

The $N$ th order PARAFAC decomposition is unique up to permutation and scaling ambiguities affecting the columns of factors matrices $\mathbf{A}^{(n)} \in \mathbb{C}^{I_{n} \times R}, n=1, \ldots, N$, if the following sufficient condition is satisfied [19]:

$$
\sum_{n=1}^{N} \kappa_{\mathbf{A}^{(n)}} \geq 2 R+N-1,
$$

where $\kappa_{\mathbf{A}^{(n)}}$ denotes the Kruskal-rank of $\mathbf{A}^{(n)}$, defined as the maximum value $\kappa$ such that any subset of $\kappa$ columns is linearly independent [20].

Throughout this work, special attention is given to dualsymmetric tensors. The PARAFAC decomposition of a given tensor $\mathscr{X} \in \mathbb{C}^{I_{1} \times \cdots \times I_{2 N}}$ of order $2 N$ is said to have dualsymmetry if defined as follows:

$$
\begin{aligned}
\mathscr{X}= & \mathscr{I}_{2 N, R} \times{ }_{1} \mathbf{A}^{(1)} \times_{2} \mathbf{A}^{(2)} \cdots \times{ }_{N} \mathbf{A}^{(N)} \times_{N+1} \mathbf{A}^{(1)^{*}} \\
& \times_{N+2} \mathbf{A}^{(2)^{*}} \cdots \times \times_{2 N} \mathbf{A}^{(N)^{*}} .
\end{aligned}
$$

Note that this definition also applies to Tucker decomposition by simply replacing the identity tensor $\mathscr{I}_{2 N, R}$ by an arbitrary core tensor $\mathscr{E}$ of order $2 N$.

\section{Signal Model}

We consider $K$ snapshots originating from the superposition of $M$ far-field narrowband signal sources sampled by a $R$ dimensional sensor array of size $N_{1} \times N_{2} \times \cdots \times N_{R}$, where $N_{r}$ is the size of the $r$ th array dimension, $r=1, \ldots, R$. The matrix $\mathbf{X} \in \mathbb{C}^{N \times K}$ collects the samples received by the sensor array, which can be factored as [10]

$$
\mathbf{X}=\left(\mathbf{A}^{(1)} \diamond \mathbf{A}^{(2)} \cdots \diamond \mathbf{A}^{(R)}\right) \mathbf{S}+\mathbf{V},
$$

where

(i) $\mathbf{A}=\mathbf{A}^{(1)} \diamond \mathbf{A}^{(2)} \cdots \diamond \mathbf{A}^{(R)} \in \mathbb{C}^{N \times M}$ is the spatial signature matrix of the $R$-D array for $r=1, \ldots, R$ and $N=\prod_{r=1}^{R} N_{r} ;$

(ii) $\mathbf{A}^{(r)}=\left[\mathbf{a}_{1}^{(r)}, \ldots, \mathbf{a}_{M}^{(r)}\right] \in \mathbb{C}^{N_{r} \times M}$ is the spatial signature matrix of the $r$ th dimension;

(iii) $\mathbf{a}_{m}^{(r)}=\left[\begin{array}{lllll}1 & e^{j \cdot \mu_{m}^{(r)}} & e^{j \cdot 2 \mu_{m}^{(r)}} & \cdots & e^{j \cdot\left(N_{r}-1\right) \mu_{m}^{(r)}}\end{array}\right]^{T} \in \mathbb{C}^{N_{r} \times 1}$ is the array response in the $r$ th dimension to the $m$ th planar wavefront $(m=1, \ldots, M)$ which is function of the spatial frequency $\mu_{m}^{(r)}$;

(iv) $\mathbf{S}=[\mathbf{s}(1), \ldots, \mathbf{s}(K)] \in \mathbb{C}^{M \times K}$ is the matrix containing the signal transmitted by the sources;

(v) $\mathbf{V}=[\mathbf{v}(1), \ldots, \mathbf{v}(K)] \in \mathbb{C}^{N \times K}$ is the additive white Gaussian noise (assumed uncorrelated to the source signals).

From (11), the sample covariance matrix $\widehat{\mathbf{R}} \in \mathbb{C}^{N \times N}$ of the signals received at the sensor array is given by

$$
\begin{aligned}
\widehat{\mathbf{R}} \triangleq & \frac{1}{K} \mathbf{X X}^{H} \approx \mathbf{A R}_{\mathbf{s}} \mathbf{A}^{H}+\sigma_{v}^{2} \mathbf{I} \\
\approx & \left(\mathbf{A}^{(1)} \diamond \cdots \diamond \mathbf{A}^{(R)}\right) \mathbf{R}_{\mathbf{s}}\left(\mathbf{A}^{(1)} \diamond \cdots \diamond \mathbf{A}^{(R)}\right)^{H} \\
& +\sigma_{v}^{2} \mathbf{I},
\end{aligned}
$$

where $\mathbf{R}_{\mathbf{s}}=(1 / K) \mathbf{S S}^{H}$ is the sample covariance matrix of the source signals and $\sigma_{v}^{2}$ is the noise variance.

\section{Tensor-Based Methods for Blind Spatial Signature Estimation}

In this section, we propose two iterative algorithms to solve the blind spatial signature estimation problem in $R-\mathrm{D}$ sensor arrays. Initially, a novel multidimensional structure is formulated from the covariance matrix of the received data. Then, an alternating least squares- (ALS-) based algorithm for a Tucker decomposition of order $2 \mathrm{~N}$ is proposed. Finally, we derive a link between the method in [12] and a covariancebased blind spatial signature estimation problem. 
4.1. Novel Covariance Tensor. With the intention of exploiting the multidimensional structure of the received signal, the noiseless sample covariance matrix (12), given by $\mathbf{R}_{\mathbf{o}}=\widehat{\mathbf{R}}-$ $\sigma_{v}^{2} \mathbf{I} \in \mathbb{C}^{N \times N}$, is interpreted as a multimode unfolding of the noiseless covariance tensor $\mathscr{R}_{\mathbf{0}} \in \mathbb{C}^{N_{1} \times N_{2} \times \cdots \times N_{R} \times N_{1} \times N_{2} \times \cdots \times N_{R}}$ of order $2 N$, defined as

$$
\begin{aligned}
\mathscr{R}_{\mathbf{o}}= & \mathscr{R}_{\mathbf{s}} \times{ }_{1} \mathbf{A}^{(1)} \times{ }_{2} \mathbf{A}^{(2)} \cdots \times{ }_{R} \mathbf{A}^{(R)} \times_{R+1} \mathbf{A}^{(1)^{*}} \\
& \times_{R+2} \mathbf{A}^{(2)^{*}} \cdots \times \times_{2 R} \mathbf{A}^{(R)^{*}}
\end{aligned}
$$

where $\mathscr{R}_{\text {s }}$ is the source covariance tensor, which has $2 R$ dimensions, each of size $M$. Note that this tensor is dualsymmetric; that is, the factor matrix related to $(R+r)$ th dimension is equal to $\mathbf{A}^{(r)^{*}}$, and $N_{r}=N_{R+r}(r=1, \ldots, R)$. The $m$ th frontal slice of $\mathscr{R}_{\mathrm{s}}$ is a diagonal matrix whose main diagonal is given by the $m$ th column of the covariance matrix $\mathbf{R}_{\mathbf{s}}$. For instance, considering $R=2$ for the sake of notation, the following expression satisfies the relationship previously cited:

$$
\mathscr{R}_{\mathbf{s}}(:,:, m, m)=\operatorname{diag}\left(\mathbf{R}_{\mathbf{s}}(:, m)\right) \quad m=1, \ldots, M,
$$

where the matrix $\mathscr{R}_{\mathrm{s}}(:,:, m, m) \in \mathbb{C}^{M \times M}$ denotes the $m$ th frontal slice of the covariance tensor $\mathscr{R}_{\text {s }}$ obtained by fixing its last two modes. The tensor $\mathscr{R}_{\mathbf{o}}$ follows a dual-symmetric Tucker decomposition of order $2 R$ with factor matrices $\mathbf{A}^{(r)}$ and $\mathbf{A}^{(r)^{*}}, r=1, \ldots, R$, and core tensor $\mathscr{R}_{\mathbf{s}}$.

Considering the case in which the sources are uncorrelated and have unitary variance, we can rewrite (13) as

$$
\begin{aligned}
\mathscr{R}_{\mathbf{o}}= & \mathscr{I}_{2 R, M} \times{ }_{1} \mathbf{A}^{(1)} \times{ }_{2} \mathbf{A}^{(2)} \cdots \times{ }_{R} \mathbf{A}^{(R)} \times{ }_{R+1} \mathbf{A}^{(1)^{*}} \\
& \times{ }_{R+2} \mathbf{A}^{(2)^{*}} \cdots \times{ }_{2 R} \mathbf{A}^{(R)^{*}}
\end{aligned}
$$

where $\mathscr{I}_{2 R, M}$ is the identity tensor of order $2 R$ in which each dimension has size $M$. In this case, the covariance tensor $\mathscr{R}_{\mathbf{o}}$ follows a dual-symmetric PARAFAC decomposition of order $2 R$.

In general, the Tucker decomposition does not impose restrictions on the core tensor structure, which makes this model more flexible. In the context of this paper, this characteristic reflects an arbitrary and unknown structure for the source's covariance $\mathbf{R}_{\mathbf{s}}$ which can also be estimated from (13). In contrast, the PARAFAC decomposition (15) denotes a particular case of the Tucker decomposition when the sources' signals are uncorrelated and the source covariance matrix is perfectly known (i.e., diagonal). In practice, this may not hold.

4.2. ALS-Tucker Algorithm. Our goal is to blindly estimate the spatial signature matrices $\mathbf{A}^{(r)}$ and $\mathbf{A}^{(r)^{*}},(r=1, \ldots, R)$ which refer to the different dimensions of the sensor array from the covariance tensor $\mathscr{R}_{\mathbf{0}}$. For the sake of simplicity, from this point on, we consider $\mathbf{A}^{(R+r)}=\mathbf{A}^{(r)^{*}}$. In matrixbased notation, the Tucker decomposition (13) allows the following factorization in terms of its factor matrices and core tensor in accordance with (5):

$$
\left[\mathscr{R}_{\mathbf{o}}\right]_{(r)}=\mathbf{A}^{(r)} \Delta^{(r)}
$$

where

$$
\begin{aligned}
\Delta^{(r)} & =\left[\mathscr{R}_{\mathbf{s}}\right]_{(r)} \\
& \cdot\left(\mathbf{A}^{(2 R)} \otimes \cdots \otimes \mathbf{A}^{(r+1)} \otimes \mathbf{A}^{(r-1)} \otimes \cdots \otimes \mathbf{A}^{(1)}\right)^{T},
\end{aligned}
$$

while $\left[\mathscr{R}_{\mathbf{o}}\right]_{(r)}$ and $\left[\mathscr{R}_{\mathbf{s}}\right]_{(r)}, r=1, \ldots, 2 R$, denote the $n$-mode unfolding of the covariance tensor $\mathscr{R}_{\mathbf{o}}$ and the core tensor $\mathscr{R}_{\text {s }}$, respectively.

From the matrix unfoldings of $\mathscr{R}_{\mathbf{0}}$, an ALS based algorithm is formulated to estimate the desired factor matrices. An estimate of the spatial signature matrix $\widehat{\mathbf{A}}^{(r)}(r=$ $1, \ldots, 2 R)$, associated with the $r$ th dimension of the covariance tensor, is obtained by solving the following least squares (LS) problem:

$$
\widehat{\mathbf{A}}^{(r)}=\arg \min _{\mathbf{A}^{(r)}}\left\|\left[\mathscr{R}_{\mathbf{o}}\right]_{(r)}-\mathbf{A}^{(r)} \Delta^{(r)}\right\|_{F}^{2},
$$

whose analytic solution is given by

$$
\widehat{\mathbf{A}}^{(r)}=\left[\mathscr{R}_{\mathbf{o}}\right]_{(r)}\left(\Delta^{(r)}\right)^{\dagger} .
$$

As discussed in Section 4.1, the Tucker decomposition does not impose restrictions on the structure of the core tensor and its estimation becomes necessary. Let $\mathbf{R}_{\mathbf{s}}$ be an unknown matrix of arbitrary structure. The following LS problem is formulated from the vectorization of the sample covariance matrix $\widehat{\mathbf{R}}$ :

$$
\operatorname{vec}\left(\widehat{\mathbf{R}}_{\mathbf{s}}\right)=\arg \min _{\mathbf{R}_{\mathbf{s}}}\left\|\operatorname{vec}(\widehat{\mathbf{R}})-\left(\mathbf{A}^{*} \otimes \mathbf{A}\right) \operatorname{vec}\left(\mathbf{R}_{\mathbf{s}}\right)\right\|_{F}^{2},
$$

from which an estimate of $\widehat{\mathbf{R}}_{\mathbf{s}}$ can be obtained as

$$
\operatorname{vec}\left(\widehat{\mathbf{R}}_{\mathbf{s}}\right)=\left(\mathbf{A}^{*} \otimes \mathbf{A}\right)^{\dagger} \operatorname{vec}(\widehat{\mathbf{R}})
$$

where $\mathbf{A}=\mathbf{A}^{(1)} \diamond \mathbf{A}^{(2)} \cdots \diamond \mathbf{A}^{(R)} \in \mathbb{C}^{N \times M}$.

Since (19) and (21) are nonlinear functions of the parameters to be estimated, the blind spatial signature estimation problem can be solved using a classical ALS iterative solution $[21,22]$. The basic idea of the algorithm is to estimate one factor matrix at each step while the others remain fixed at the values obtained in previous steps. This procedure is repeated until convergence. The proposed generalized ALS-Tucker algorithm for $R$-D sensor arrays is summarized in Algorithm 1.

In this approach the factor matrices are treated as independent variables; that is, the dual-symmetry property of the covariance tensor is not exploited. In this case, a final estimate of the spatial signature matrix associated with the $r$ th dimension of the array is given by

$$
\widehat{\mathbf{A}}_{\text {final }}^{(r)}=\frac{\widehat{\mathbf{A}}^{(r)}+\widehat{\mathbf{A}}^{(R+r)^{*}}}{2} .
$$

4.3. ALS-ProKRaft Algorithm. In this section, a link is established between the ALS-ProKRaft algorithm proposed initially in [12] and blind spatial signature estimation in 
(1) Initialize $\widehat{\mathbf{A}}^{(r)} \in \mathbb{C}^{N_{r} \times M}$ for $r=2, \ldots, 2 R$ and the core tensor $\widehat{\mathscr{R}}_{\mathrm{s}}$ randomly.

(2) for $r=1, \ldots, 2 R$ do

According to (19), obtain an estimate for the matrix $\widehat{\mathbf{A}}^{(r)}$ : $\widehat{\mathbf{A}}^{(r)}=[\mathscr{R}]_{(r)}\left(\Delta^{(r)}\right)^{\dagger}$.

Note. The matrix $\Delta^{(r)}$, described in (17), is updated end by fixing $\widehat{\mathbf{A}}^{(r)}$ calculated previously.

(3) According to (21), obtain an estimate for $\widehat{\mathbf{R}}_{\boldsymbol{s}}$ : $\operatorname{vec}\left(\widehat{\mathbf{R}}_{\mathbf{s}}\right)=\left(\mathbf{A}^{*} \otimes \mathbf{A}\right)^{\dagger} \operatorname{vec}(\widehat{\mathbf{R}}) ;$

$\widehat{\mathbf{R}}_{\mathbf{s}}=\operatorname{unvec}_{M \times M}\left(\operatorname{vec}\left(\widehat{\mathbf{R}}_{\mathbf{s}}\right)\right)$.

(4) Using (14), reconstruct the core tensor $\widehat{\mathscr{R}}_{\mathrm{s}}$ from $\widehat{\mathbf{R}}_{\mathbf{s}}$.

(5) Repeat Steps (2)-(4) until convergence.

Algorithm 1: Summary of the ALS-Tucker algorithm.

array signal processing. The main idea behind this algorithm is to exploit the dual-symmetry property of the PARAFAC decomposition described in (15). Next, the ALS-ProKRaft algorithm is formulated in the context of this work. A more detailed description of the method can be found in the original reference.

The multimode unfolding of the PARAFAC decomposition in (15) can be rewritten as

$$
\mathbf{R}_{\mathrm{mm}}=\left(\mathbf{A}^{(1)} \diamond \cdots \diamond \mathbf{A}^{(R)}\right)\left(\mathbf{A}^{(1)} \diamond \cdots \diamond \mathbf{A}^{(R)}\right)^{H},
$$

which assumes the following factorization:

$$
\mathbf{R}_{\mathrm{mm}}=\mathbf{R}_{\mathrm{mm}}^{1 / 2} \cdot\left(\mathbf{R}_{\mathrm{mm}}^{1 / 2}\right)^{H},
$$

where $\mathbf{R}_{\mathrm{mm}}^{1 / 2} \in \mathbb{C}^{N \times M}$ can be obtained from the singular value decomposition of $\mathbf{R}_{\mathrm{mm}}$ given by

$$
\mathbf{R}_{\mathrm{mm}}=\mathbf{U} \cdot \boldsymbol{\Sigma} \cdot \mathbf{V}^{H}
$$

obeying the following structure:

$$
\mathbf{R}_{\mathrm{mm}}^{1 / 2}=\mathbf{U}^{[M]} \cdot \boldsymbol{\Sigma}^{[M]} \cdot \mathbf{T}^{H}=\left(\mathbf{A}^{(1)} \diamond \cdots \diamond \mathbf{A}^{(R)}\right),
$$

where $\mathbf{U}^{[M]} \in \mathbb{C}^{N \times M}$ is formed by the first $M$ columns of $\mathbf{U}$ and $\Sigma^{[M]} \in \mathbb{C}^{M \times M}$ is a diagonal matrix which contains the $M$ dominant singular values of $\mathbf{R}_{\mathrm{mm}}$. The matrix $\mathbf{T}$ represents a unitary rotation factor.

Equation (26) describes an orthogonal Procrustes problem (OPP) [23], in which $\mathbf{T}$ is a transformation matrix that maps $\mathbf{U}^{[M]} \cdot \Sigma^{[M]}$ to $\left(\mathbf{A}^{(1)} \diamond \cdots \diamond \mathbf{A}^{(R)}\right)$ such that $\mathbf{U}^{[M]} \cdot \Sigma^{[M]}$. $\mathbf{T}^{H}=\left(\mathbf{A}^{(1)} \diamond \cdots \diamond \mathbf{A}^{(R)}\right)$. An efficient estimate for $\mathbf{T}$ is obtained minimizing the Frobenius norm of the residual error:

$$
\arg \min _{\mathbf{T}}\left\|\mathbf{U}^{[M]} \cdot \mathbf{\Sigma}^{[M]} \cdot \mathbf{T}^{H}-\left(\mathbf{A}^{(1)} \diamond \cdots \diamond \mathbf{A}^{(R)}\right)\right\|_{F} .
$$

This problem can be solved using a change of basis from the singular value decomposition of the matrix

$$
\left(\mathbf{A}^{(1)} \diamond \cdots \diamond \mathbf{A}^{(R)}\right)^{H} \cdot \mathbf{U}^{[M]} \cdot \mathbf{\Sigma}^{[M]}=\mathbf{U}_{P} \cdot \boldsymbol{\Sigma}_{P} \cdot \mathbf{V}_{P}^{H},
$$

which leads to the following solution [23]:

$$
\widehat{\mathbf{T}}=\mathbf{U}_{P} \cdot \mathbf{V}_{P}^{H} .
$$

From (26) and (29), an ALS-based iterative algorithm is formulated to estimate the spatial signature matrices from the PARAFAC decomposition (15). Firstly, individual estimates for each factor matrix $\widehat{\mathbf{A}}^{(r)}, r=1, \ldots, R$, are obtained by applying the multidimensional LS Khatri-Rao factorization (LS-KRF) algorithm on the composite spatial signature matrix $\widehat{\mathbf{A}}=\widehat{\mathbf{A}}^{(1)} \diamond \widehat{\mathbf{A}}^{(2)} \cdots \diamond \widehat{\mathbf{A}}^{(R)}$. Then, the matrix $\widehat{\mathbf{T}}$ is obtained from (29). For more details and access to the pseudocode of this algorithm, we refer the interested reader to [12]. The ALS-ProKRaft algorithm for blind spatial signature estimation in $R-\mathrm{D}$ sensor arrays is summarized in Algorithm 2.

Note that when compared with conventional ALS-based PARAFAC solutions [21] formulated from the unfolding matrices (8), the ALS-ProKRaft algorithm becomes preferred since only half of the factors matrices needs to be estimated by exploiting the dual-symmetry property of the covariance tensor. This generally leads to a fast convergence rate of the algorithm.

\section{Spatial Signature Estimation in $L$-Shaped Sensor Arrays}

In this section, the blind spatial signature estimation problem is formulated for $L$-shaped array configuration. Considering that the receiver array is divided into smaller subarrays, the Tucker decomposition of a fourth-order tensor is formulated from the sample cross-correlation matrix of the data received by the different subarrays. From this multidimensional structure the proposed generalized ALS-Tucker algorithm previously presented in Section 4.2 can be used to estimate the source's spatial signatures.

5.1. Cross-Correlation Tensor for L-Shaped Sensor Arrays. In this approach, we consider an $L$-shaped sensor array equipped with $N_{1}+N_{2}-1$ sensors positioned in the $x-z$ 
$i=0$; Initialize $\widehat{\mathbf{T}}_{(i=0)}=\mathbf{I}_{M}$.

(1) According to (25), obtain $\mathbf{U}^{[M]}$ and $\boldsymbol{\Sigma}^{[M]}$ from the SVD

of the multimode unfolding matrix $\mathbf{R}_{\mathrm{mm}}$.

(2) $i=i+1$;

(3) According to (26), obtain estimates for $\widehat{\mathbf{A}}_{(i)}^{(r)}$ for $r=1, \ldots, R$ by applying the multidimensional LS-KRF algorithm on $\mathbf{U}^{[M]} \cdot \Sigma^{[M]} \cdot \widehat{\mathbf{T}}_{(i-1)}^{H}$.

(4) According to (29), compute the SVD for the matrix $\left(\widehat{\mathbf{A}}_{(i)}^{(1)} \diamond \cdots \diamond \widehat{\mathbf{A}}_{(i)}^{(R)}\right)^{H} \cdot \mathbf{U}^{[M]} \cdot \Sigma^{[M]}=\mathbf{U}_{P} \cdot \boldsymbol{\Sigma}_{P} \cdot \mathbf{V}_{P}^{H}$ and obtain $\widehat{\mathbf{T}}_{(i)}=\mathbf{U}_{P} \cdot \mathbf{V}_{P}^{H}$.

(5) Repeat Steps (2)-(4) until convergence.

Algorithm 2: Summary of the ALS-ProKRaft algorithm.

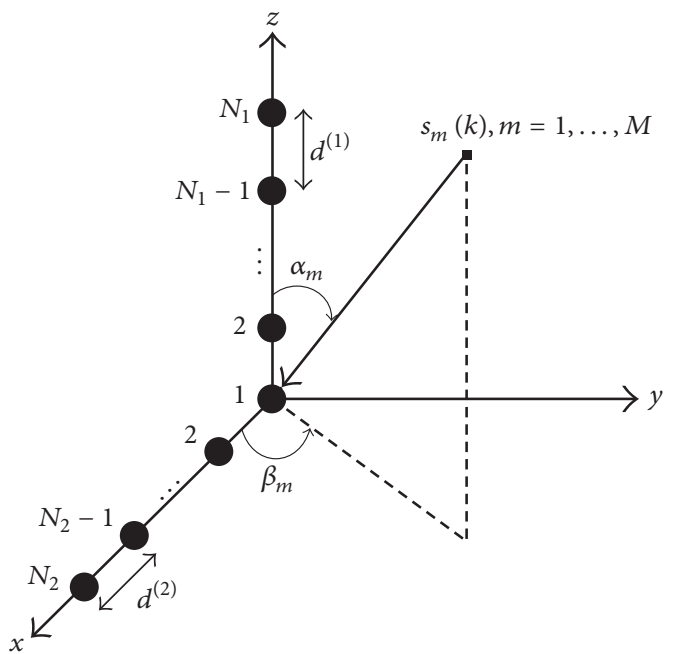

FIGURE 1: $L$-shaped array configuration with $N_{1}+N_{2}-1$ sensors. The distance between the sensors in the $z$-axis is $d^{(1)}$ while the distance between the sensors in the $x$-axis is $d^{(2)}$. The $m$ th wavefront has elevation and azimuth angles equal to $\alpha_{m}$ and $\beta_{m}$, respectively.

plane, as illustrated in Figure 1. Each linear array contains $N_{1}$ and $N_{2}$ sensors equally spaced at distances $d^{(1)}$ and $d^{(2)}$, respectively. We consider that each linear array is divided into $P$ and $W$ smaller subarrays, respectively. Each subarray contains $N_{1}^{(\mathrm{sub})}=N_{1}-P+1$ and $N_{2}^{(\text {sub })}=N_{2}-W+1$ sensors. The signal received at the $p$ th subarray, for $p=1, \ldots, P$, is given by

$$
\mathbf{X}_{p}^{(1)}=\mathbf{A}_{\mathbf{s}}^{(1)} \mathbf{D}_{p}\left(\Phi^{(1)}\right) \mathbf{S}+\mathbf{V}_{p}^{(1)} \in \mathbb{C}^{N_{1}^{(\mathrm{sub})} \times K},
$$

and the signal received at the $w$ th subarray, $w=1, \ldots, W$, is given by

$$
\mathbf{X}_{w}^{(2)}=\mathbf{A}_{\mathbf{s}}^{(2)} \mathbf{D}_{w}\left(\Phi^{(2)}\right) \mathbf{S}+\mathbf{V}_{w}^{(2)} \in \mathbb{C}^{N_{2}^{(\mathrm{sub})} \times K},
$$

where

(i) $\mathbf{A}_{\mathrm{s}}^{(r)} \in \mathbb{C}^{N_{r}^{(\text {sub })} \times M}$ is the spatial signature matrix of the first subarray (or reference subarray) for the $r$ th array dimension, $r=1,2$; (ii) $\mathbf{D}_{p}\left(\Phi^{(1)}\right)$ and $\mathbf{D}_{w}\left(\Phi^{(2)}\right)$ are diagonal matrices whose main diagonal is given by the $p$ th and $w$ th row of the matrices $\boldsymbol{\Phi}^{(1)} \in \mathbb{C}^{P \times M}$ and $\Phi^{(2)} \in \mathbb{C}^{W \times M}$, respectively.

The rows of $\boldsymbol{\Phi}^{(1)}$ and $\boldsymbol{\Phi}^{(2)}$ capture the delays suffered by the signals impinging the $p$ th and $w$ th subarrays with respect to the reference subarray, which are defined based on the following spatial frequencies:

$$
\begin{aligned}
& \mu_{m}^{(1)}=\frac{2 \pi \cdot d^{(1)} \cdot \cos \alpha_{m}}{\lambda}, \\
& \mu_{m}^{(2)}=\frac{2 \pi \cdot d^{(2)} \cdot \sin \alpha_{m} \cdot \cos \beta_{m}}{\lambda},
\end{aligned}
$$

where $\alpha_{m}$ and $\beta_{m}$ are the azimuth and elevation angles of the $m$ th source, respectively.

From (30) and (31), let us introduce the following extended data matrices:

$$
\begin{aligned}
& \mathbf{X}^{(1)}=\left[\mathbf{X}_{1}^{(1)}, \ldots, \mathbf{X}_{P}^{(1)}\right]^{T} \in \mathbb{C}^{N_{1}^{(\text {sub })} P \times K}, \\
& \mathbf{X}^{(2)}=\left[\mathbf{X}_{1}^{(2)}, \ldots, \mathbf{X}_{W}^{(2)}\right]^{T} \in \mathbb{C}^{N_{2}^{(\text {sub })} W \times K},
\end{aligned}
$$

or, more compactly,

$$
\mathbf{X}^{(r)}=\left(\Phi^{(r)} \diamond \mathbf{A}_{\mathbf{s}}^{(r)}\right) \mathbf{S}+\mathbf{V}^{(r)}, \quad r=1,2 .
$$

In contrast with (12), in this approach we shall work with the following sample cross-correlation matrix:

$$
\widehat{\mathbf{R}}=\left(\boldsymbol{\Phi}^{(1)} \diamond \mathbf{A}_{\mathbf{s}}^{(1)}\right) \mathbf{R}_{\mathbf{s}}\left(\boldsymbol{\Phi}^{(2)} \diamond \mathbf{A}_{\mathbf{s}}^{(2)}\right)^{H}+\sigma_{v}^{2} \mathbf{I} .
$$

As mentioned in Section 4.1, we can see that the noiseless term in (35) denotes a multimode unfolding of the following cross-correlation tensor of size $N_{1}^{\text {(sub) }} \times P \times N_{2}^{\text {(sub) }} \times W$ :

$$
\mathscr{R}_{\mathrm{o}}=\mathscr{R}_{\mathrm{s}} \times{ }_{1} \mathrm{~A}_{\mathrm{s}}^{(1)} \times_{2} \Phi^{(1)} \times{ }_{3} \mathrm{~A}_{\mathrm{s}}^{(2)^{*}} \times_{4} \Phi^{(2)^{*}},
$$


where $\mathscr{R}_{\mathrm{s}} \in \mathbb{C}^{M \times M \times M \times M}$. In this modeling, the tensor $\mathscr{R}_{\mathbf{o}}$ follows a fourth-order Tucker decomposition. By analogy with (4), we deduce the following correspondences:

$$
\begin{aligned}
& \left(I_{1}, I_{2}, I_{3}, I_{4}\right) \longleftrightarrow\left(N_{1}^{(\mathrm{sub})}, P, N_{2}^{(\mathrm{sub})}, W\right) \\
& \left(R_{1}, R_{2}, R_{3}, R_{4}\right) \longleftrightarrow M \\
& \left(\mathscr{G}, \mathbf{A}^{(1)}, \mathbf{A}^{(2)}, \mathbf{A}^{(3)}, \mathbf{A}^{(4)}\right) \\
& \longleftrightarrow\left(\mathscr{R}_{\mathbf{s}}, \mathbf{A}_{\mathbf{s}}^{(1)}, \Phi^{(1)}, \mathbf{A}_{\mathbf{s}}^{(2)^{*}}, \boldsymbol{\Phi}^{(2)^{*}}\right) .
\end{aligned}
$$

The spatial signatures of the sources can be estimated from (36) by using the proposed generalized ALS-Tucker algorithm. Note that, in this case, the ALS-Tucker algorithm is simplified to a fourth-order tensor input.

For all the previously proposed algorithms, the final estimates for the spatial signature matrices are obtained when the convergence is declared. A usually adopted criterion for convergence is defined as $\left|e_{(i)}-e_{(i-1)}\right| \leq 10^{-6}$, where $e_{(i)}$ denotes the residual error of the $i$ th iteration, defined as

$$
e_{(i)}=\left\|\mathscr{R}-\widehat{\mathscr{R}}_{(i)}\right\|_{F}^{2},
$$

where $\mathscr{R}=\mathscr{R}_{\mathbf{o}}+\mathscr{V}$ is a noisy version of $\mathscr{R}, \mathscr{V}$ is an additive complex-valued white Gaussian noise tensor, and $\widehat{\mathscr{R}}_{(i)}$ is the covariance tensor reconstructed from the estimated factor matrices and core tensor. Since the ALS-ProKRaft algorithm exploits the dual-symmetry property of the data tensor the procedure in (22) is not necessary.

5.2. Estimation of the Spatial Frequencies. After the estimation of the spatial signatures matrices $\widehat{\mathbf{A}}_{\text {final }}^{(r)}, r=1, \ldots, R$, the final step is to estimate the spatial frequencies of the sources $\widehat{\mu}_{m}^{(r)}, m=1, \ldots, M$. The final estimates can be computed from the average over the values obtained in each row of $\widehat{\mathbf{A}}_{\text {final }}^{(r)}$ as follows:

$$
\widehat{\mu}_{m}^{(r)}=\frac{1}{N_{r}-1} \sum_{n=2}^{N_{r}} \frac{\arg \left\{\widehat{\mathbf{A}}_{\text {final }}^{(r)}(n, m)\right\}}{n-1} .
$$

\section{Computational Complexity}

In the following, we discuss the computational complexity of the iterative ALS-Tucker and ALS-ProKRaft algorithms. For the sake of simplicity, the computational complexity of the proposed methods is described in terms of the computational cost of the matrix SVD. For a matrix of size $I_{1} \times I_{2}$ the number of floating-point operations associated with the SVD computation is $\mathcal{O}\left(I_{1} \cdot I_{2} \cdot \min \left(I_{1} \cdot I_{2}\right)\right)$ [24]. The computational complexity of one Tucker iteration refers to the cost associated with the SVD used to calculate the matrix pseudoinverses in the least squares problems (18) and (20). The overall computational complexity per iteration of the ALS-Tucker algorithm equals the complexity of $2 R$ matrix SVDs associated with each estimated factor matrix according to (19) plus the complexity of one additional matrix SVD associated with the estimated core tensor according to (21).
The overall computational cost per iteration of the ALSProKRaft algorithm equals the complexity of $M(R-1)$ matrix SVDs associated with the application of the multidimensional LS-KRF algorithm in (26) plus the complexity of one additional matrix SVD associated with the update of the unknown unitary rotation factor matrix $\mathbf{T}$ according to (29).

\section{Advantages and Disadvantages of the Proposed Methods}

In this section, we discuss the advantages and disadvantages of the proposed methods to blind spatial signatures estimation in $R$-D sensor arrays. As previously stated in Section 4.3, the ALS-ProKRaft algorithm works on the assumption that the sample covariance matrix of the sources $\mathbf{R}_{\mathbf{s}}$ is perfectly known and diagonal. However, this is only true in the asymptotic case when a sufficiently large number of snapshots is assumed (i.e., $K \rightarrow \infty$ ), as well as when the source signals are perfectly uncorrelated. In practice, this assumption is not guaranteed. On the other hand, the ALS-Tucker algorithm previously formulated in Section 4.2 naturally captures any structure for the sources covariance into the core tensor $\mathscr{R}_{\mathrm{s}}$. Therefore, the assumption of uncorrelated source signals is not necessary for the ALS-Tucker algorithm, making it able to operate in scenarios where the source covariance structure is unknown and arbitrary (nondiagonal). Such scenarios occur, for instance, when the sample covariance is computed from a limited number of snapshots.

In contrast, the ALS-Tucker algorithm does not exploit the dual-symmetry property of the data covariance tensor and all factor matrices need to be estimated as independent variables. However, in the ALS-ProKRaft algorithm, only half of the factor matrices are estimated by exploiting the dualsymmetry property of the covariance tensor. Therefore, the ALS-ProKRaft algorithm is more computationally attractive than the ALS-Tucker algorithm. When compared with the state-of-the-art matrix-based algorithms such as MUSIC, ESPRIT, and Propagator Method, the proposed tensor-based algorithms have the advantage of fully exploiting the multidimensional nature of the received signal in less specific scenarios, which leads to more accurate estimates. For instance, the ESPRIT algorithm was formulated for sensor arrays that obey the shift invariance property. On the other hand, the MUSIC algorithm has high computational complexity due to the search of parameters in the spatial spectrum.

\section{Simulation Results}

In the following, simulation results and performance evaluations of the ALS-Tucker and ALS-ProKRaft algorithms for $R$-D sensor arrays are presented. This section is divided into two parts. Firstly, the results related to Section 4 are presented and discussed. Then, the same is done for the $L$-shaped array approach presented in Section 5. Results are obtained from an average of 1000 independent Monte Carlo runs. In the first part of this section, we consider a uniform rectangular array (URA) positioned on the $x$ - $z$ plane. The $m$ th wavefront 


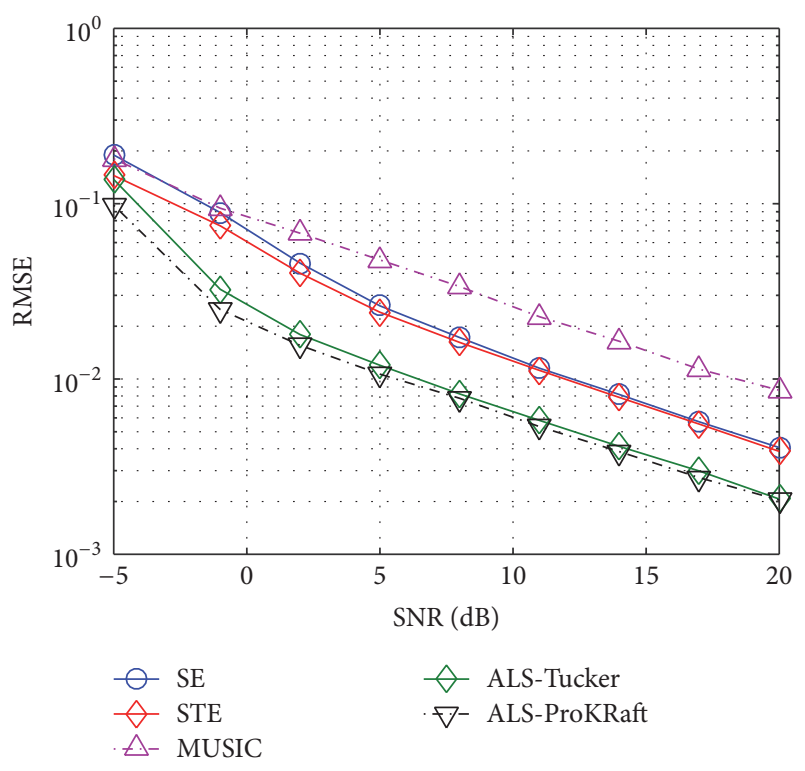

FIgUre 2: Total RMSE versus SNR for $N=64$ sensors, $K=10$ samples, and DoAs: $\left\{30^{\circ}, 55^{\circ}\right\}$ and $\left\{45^{\circ}, 60^{\circ}\right\}$ for Hadamard sequences.

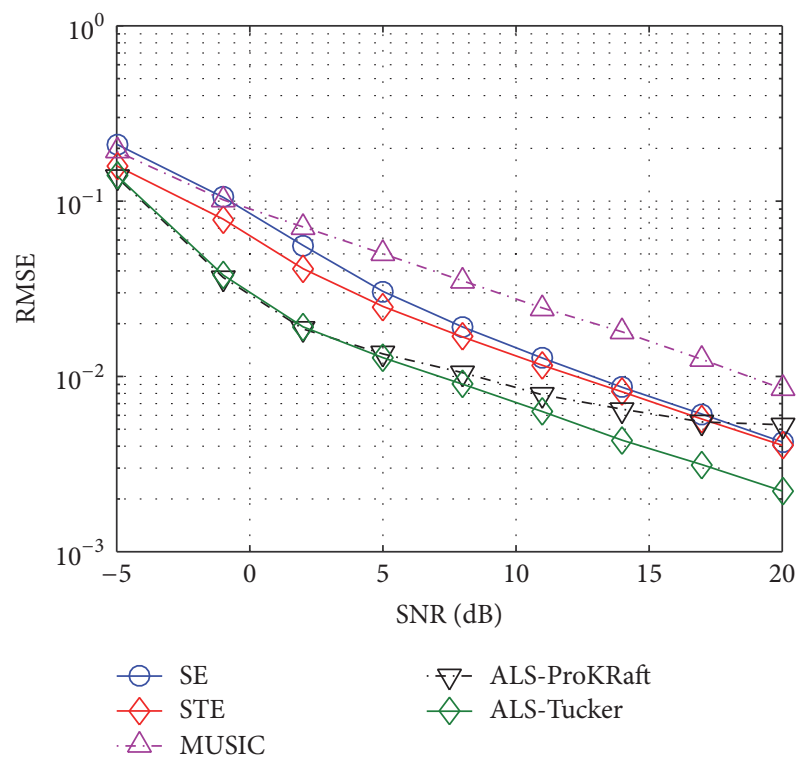

Figure 3: Total RMSE versus SNR for $N=64$ sensors, $K=10$ samples, and DoAs: $\left\{30^{\circ}, 55^{\circ}\right\}$ and $\left\{45^{\circ}, 60^{\circ}\right\}$ for BPSK sequences.

has direction of arrival $\left\{\alpha_{m}, \beta_{m}\right\}_{m=1}^{M}$, where $\alpha_{m}$ and $\beta_{m}$ are elevation and azimuth angles, respectively.

In Figures 2 and 3, the performance is measured in terms of the root mean square error (RMSE) of the estimated spatial frequencies $\widehat{\mu}_{m}^{(r)}$ in terms of Signal to Noise Ratio (SNR). The relations between directions of arrival and spatial frequencies are given by (32), where $d^{(r)}$ denotes the distance between sensors in the $r$ th array dimension, which is assumed here equal to $\lambda / 2$. In Figure 2, we consider Hadamard sequences for the sources, while in Figure 3 we consider BPSK modulated sources. In both cases, we have $N=64$ sensors

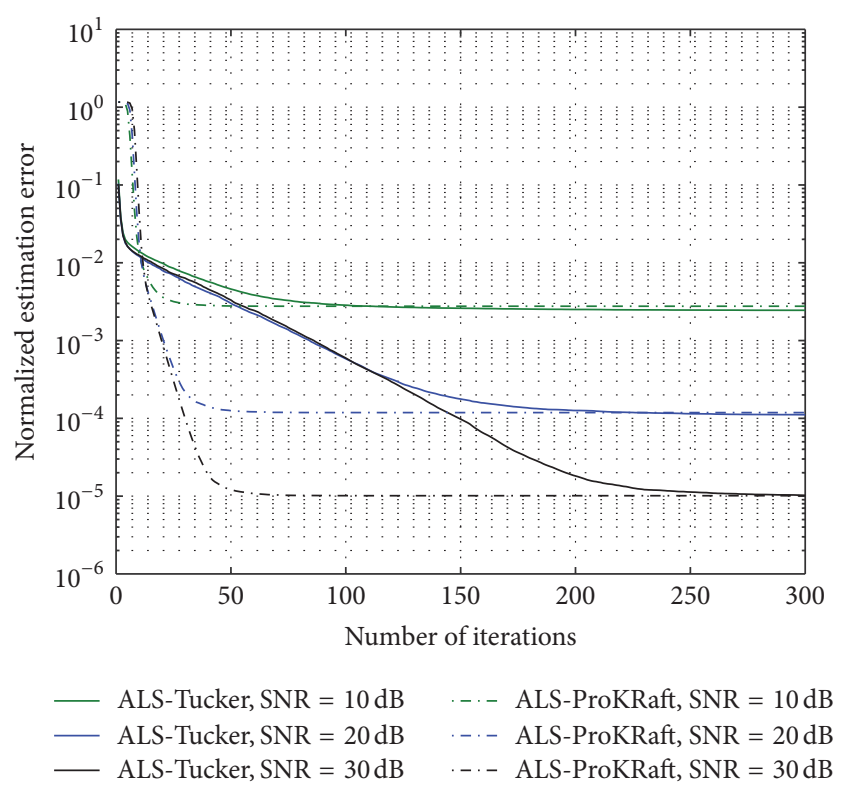

FIgURE 4: Convergence of the ALS-Tucker and ALS-ProKRaft algorithms.

(i.e., $N_{1}$ and $N_{2}$ equal to 8 ) and the sample covariance matrix of the received data (12) is calculated from a reduced number $K=10$ of samples. The total RMSE is defined as

$$
\text { RMSE }=\sqrt{E\left\{\sum_{r=1}^{R} \sum_{m=1}^{M}\left(\mu_{m}^{(r)}-\widehat{\mu}_{m}^{(r)}\right)^{2}\right\}} .
$$

From Figure 2, it can be seen that both ALS-Tucker and ALS-ProKRaft algorithms have similar performances in terms of RMSE, when Hadamard sequences are considered. In contrast, in Figure 3, when BPSK symbols are considered, a floor is exhibited by the ALS-ProKraft algorithm for high SNR values. This behavior occurs due to the modeling errors in the core tensor of the PARAFAC decomposition, which in turn arises due to the nonorthogonality of the source signals, resulting in a nondiagonal sample covariance matrix of the sources in this case. Note that in the ALS-Tucker algorithm the covariance matrix of the sources possess an arbitrary and unknown structure which discards possible constraints to the source signals. This difference makes the Tucker decomposition approach more attractive in those practical scenarios in which source uncorrelatedness is not guaranteed. When compared to matrix-based standard ESPRIT (SE) [2], matrixbased MUSIC algorithm to planar array configuration [25], and tensor-based standard ESPRIT (STE) [10], the proposed algorithms have improved accuracy in all the considered scenarios.

Figure 4 shows the convergence performance of the iterative algorithms. In this experiment, the median values of the normalized estimation error $e_{(i)} / N^{(2 R)}$ are plotted in terms of the number of iterations for different SNR. It is noteworthy that the ALS-ProKRaft algorithm has a faster convergence compared to the ALS-Tucker algorithm. This 


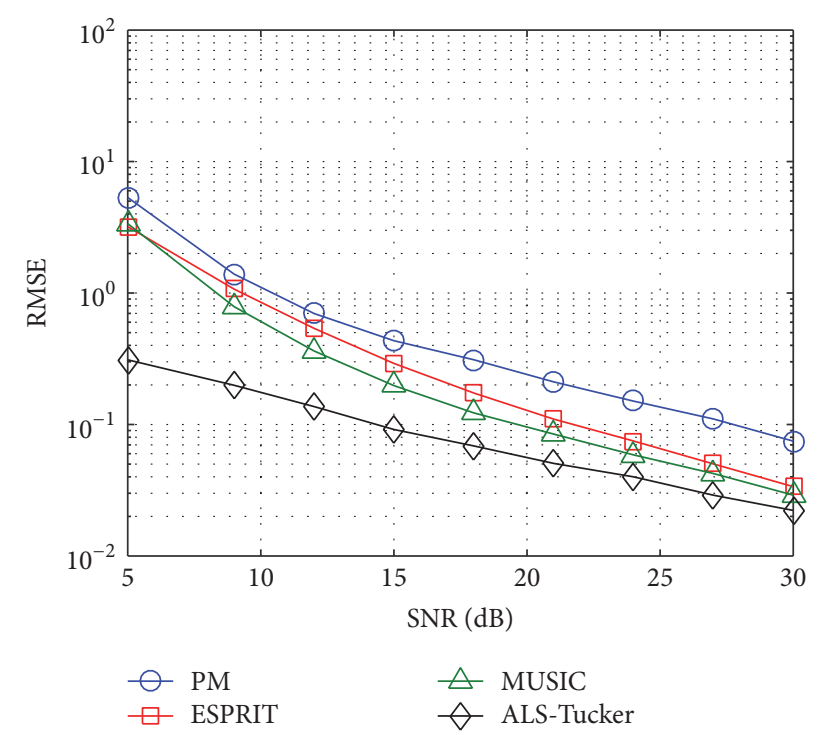

Figure 5: Total RMSE versus SNR for $N=13$ sensors, $K=500$ samples, and DoAs: $\left\{30^{\circ}, 45^{\circ}\right\}$ and $\left\{50^{\circ}, 55^{\circ}\right\}$.

behavior is expected since ALS-ProKRaft exploits the dualsymmetry property of the data tensor, which results in estimating half as many factor matrices compared to the ALSTucker approach.

In the second part of this section, we consider a $L$-shaped configuration array. In Figure 5, we set $N=13$ sensors (i.e., $N_{1}$ and $N_{2}$ equal to 7) and $K=500$ samples. Each uniform linear array is divided into $P=2$ and $W=2$ subarrays, respectively. In this experiment, the performance of the proposed ALS-Tucker algorithm is compared to the state-of-the-art matrix-based methods, namely, Propagator Method (PM) [26], MUSIC [27], and ESPRIT [28], all of them originally formulated for $L$-shaped arrays. Note that the ALSTucker algorithm presents an improved performance over its competitors, with more evidenced gains in the low-tomedium SNR range. For high SNR values, the performance of the MUSIC method comes close to that of our proposal. However, the ALS-Tucker algorithm dispenses any estimation procedure via bidimensional peak search as occurs with MUSIC, being the former more computationally attractive.

Figure 6 shows the performance of the ALS-Tucker by assuming different number of sensors. In this experiment, we consider $K=500$ samples. We can observe a better performance in terms of RMSE when the number sensors of the $L$-shaped array is increased. This is valid for all the simulated SNR values.

In Figure 7, we analyze the influence of the number $K$ of samples on the performance of the ALS-Tucker algorithm. This experiment considers the same parameters as the experiment of Figure 5, except the SNR value that is assumed fixed at $20 \mathrm{~dB}$ and the number of samples that varies between 50 and 3000 . First, we can see that the performance of the algorithms improves by increasing the number of samples collected by sensor array, as expected. However, similar to Figure 5 the

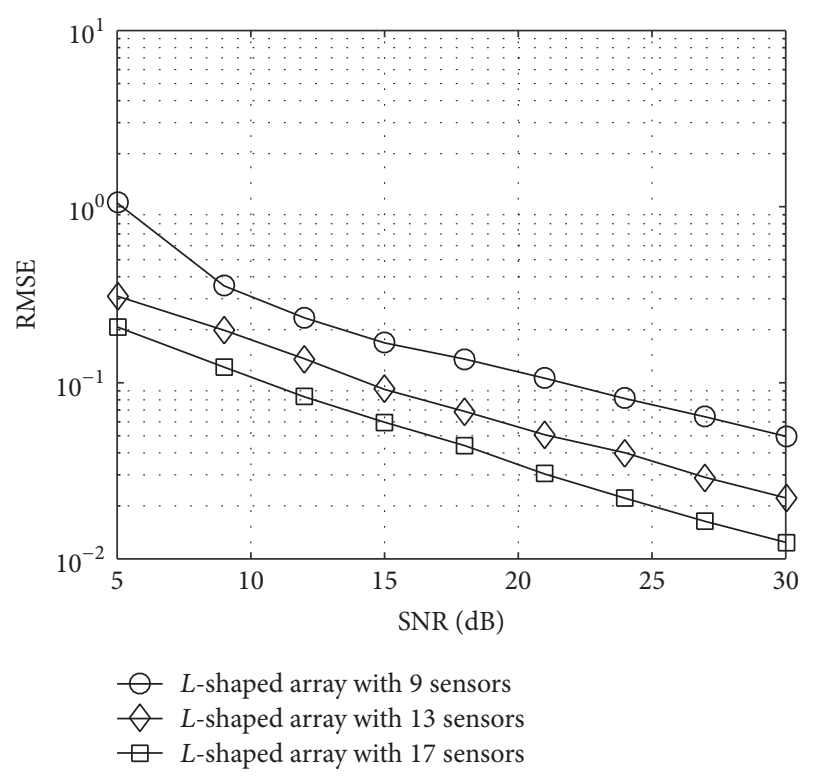

FIGURE 6: Total RMSE versus SNR (performance of the ALS-Tucker algorithm for different number of sensors).

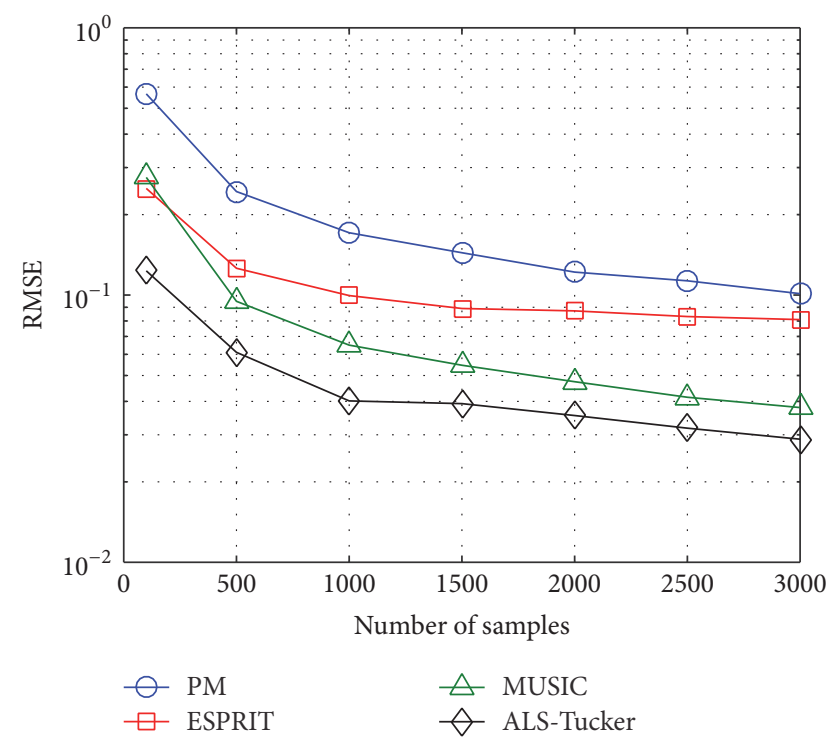

FIgURE 7: Total RMSE versus number of samples.

proposed ALS-Tucker algorithm outperforms the state-ofthe-art PM, ESPRIT, and MUSIC methods.

\section{Conclusion}

In this paper, two tensor-based approaches based on the Tucker and PARAFAC decompositions have been formulated to solve the blind spatial signatures estimation problem in multidimensional sensor arrays. First, we have proposed a covariance-based generalization of the Tucker decomposition for $R$-D sensor arrays. Then, a link between the ALS-ProKRaft algorithm and covariance-based array signal processing for blind spatial signatures estimation has been 
established. As another contribution, we have formulated a cross-correlation-based fourth-order Tucker decomposition which makes the proposed ALS-Tucker algorithm applicable in scenarios composed by $L$-shaped array configurations. The two proposed tensor methods differ in the structure assumed for the source covariance. It is worth pointing out that, in realistic scenarios, when the received covariance matrix is calculated from a reduced number of samples, or snapshots, the ALS-Tucker algorithm becomes preferable since it operates with an arbitrary and unknown structure for the covariance of the source signals. In contrast, when the sources can be assumed to be uncorrelated, we can achieve improved performance by exploiting the dualsymmetry property of the covariance tensor, which makes the ALS-ProKRaft algorithm preferable since it provides good estimation accuracy with a smaller number of ALS iterations. Finally, when compared with other state-of-the-art matrixbased and tensor-based techniques, the proposed tensorbased iterative algorithms have shown their effectiveness with remarkable gains in terms of estimation error.

\section{Competing Interests}

The authors declare that there is no conflict of interests regarding the publication of this paper.

\section{Acknowledgments}

The authors would like to thank the Fundação Cearense de Apoio ao Desenvolvimento Científico e Tecnológico (FUNCAP), the Coordenação de Aperfeiçoamento de Pessoal de Nível Superior (CAPES) under the PVE Grant no. 88881.030392/2013-01, the productivity Grant no. 303905/ 2014-0, the postdoctoral scholarship abroad (PDE) no. 207644/2015-2, and the Program Science without Borders, Aerospace Technology supported by CNPq and CAPES for the postdoctoral scolarship abroad (PDE) no. 207644/20152.

\section{References}

[1] R. O. Schmidt, "Multiple emitter location and signal parameter estimation," IEEE Transactions on Antennas and Propagation, vol. 34, no. 3, pp. 276-280, 1986.

[2] R. Roy and T. Kailath, "ESPRIT-estimation of signal parameters via rotational invariance techniques," IEEE Transactions on Acoustics, Speech, and Signal Processing, vol. 37, no. 7, pp. 984995, 1989.

[3] J. P. C. L. da Costa, F. Roemer, M. Haardt, and R. T. de Sousa Jr., "Multi-dimensional model order selection," EURASIP Journal on Advances in Signal Processing, vol. 2011, article 26, 2011.

[4] F. Roemer, Advanced algebraic concepts for efficient multichannel signal processing [Ph.D. thesis], Ilmenau University of Technology, Ilmenau, Germany, 2013.

[5] J. P. C. L. Da Costa, K. Liu, H. Cheung So, S. Schwarz, M. Haardt, and F. Römer, "Multidimensional prewhitening for enhanced signal reconstruction and parameter estimation in colored noise with Kronecker correlation structure," Signal Processing, vol. 93, no. 11, pp. 3209-3226, 2013.
[6] J. P. C. L. da Costa, Parameter Estimation Techniques for MultiDimensional Array Signal Processing, Shaker Publisher, Aachen, Germany, 2010.

[7] R. Harshman, "Foundations of the PARAFAC procedure: models and conditions for an explanatory multimodal factor analysis," UCLA Working Papers in Phonetics, vol. 16, no. 10, pp. $1-84,1970$.

[8] R. A. Harshman and M. E. Lundy, "Uniqueness proof for a family of models sharing features of Tucker's three-mode factor analysis and PARAFAC/CANDECOMP," Psychometrika. A Journal of Quantitative Psychology, vol. 61, no. 1, pp. 133-154, 1996.

[9] N. D. Sidiropoulos, R. Bro, and G. B. Giannakis, "Parallel factor analysis in sensor array processing," IEEE Transactions on Signal Processing, vol. 48, no. 8, pp. 2377-2388, 2000.

[10] M. Haardt, F. Roemer, and G. Del Galdo, "Higher-order SVDbased subspace estimation to improve the parameter estimation accuracy in multidimensional harmonic retrieval problems," IEEE Transactions on Signal Processing, vol. 56, no. 7, pp. 31983213, 2008.

[11] J. P. C. L. Da Costa, F. Roemer, M. Weis, and M. Haardt, "Robust R-D parameter estimation via closed-form PARAFAC," in Proceedings of the International ITG Workshop on Smart Antennas (WSA '10), pp. 99-106, Bremen, Germany, February 2010.

[12] M. Weis, F. Roemer, M. Haardt, and P. Husar, "Dual-symmetric parallel factor analysis using procrustes estimation and KhatriRao factorization," in Proceedings of the 20th EUSIPCO European Signal Processing Conference, Bucharest, Romania, August 2012.

[13] F. Roemer and M. Haardt, "Tensor-based channel estimation (TENCE) for two-way relaying with multiple antennas and spatial reuse," in Proceedings of the IEEE International Conference on Acoustics, Speech, and Signal Processing (ICASSP '09), Taipei, Taiwan, April 2009.

[14] P. R. B. Gomes, A. L. F. de Almeida, and J. P. C. L. da Costal, "Fourth-order tensor method for blind spatial signature estimation," in Proceedings of the IEEE International Conference on Acoustics, Speech, and Signal Processing (ICASSP '14), pp. 2992-2996, IEEE, Florence, Italy, May 2014.

[15] L. R. Tucker, "Implications of factor analysis of three-way matrices for measurement of change," in Problems in Measuring Change, C. W. Harris, Ed., pp. 122-137, University of Wisconsin Press, 1963.

[16] T. G. Kolda and B. W. Bader, "Tensor decompositions and applications," SIAM Review, vol. 51, no. 3, pp. 455-500, 2009.

[17] A. Cichocki, D. Mandic, L. De Lathauwer et al., "Tensor decompositions for signal processing applications: from twoway to multiway component analysis," IEEE Signal Processing Magazine, vol. 32, no. 2, pp. 145-163, 2015.

[18] J. M. F. Ten Berge and A. K. Smilde, "Non-triviality and identification of a constrained Tucker3 analysis," Journal of Chemometrics, vol. 16, no. 12, pp. 609-612, 2002.

[19] N. D. Sidiropoulos and R. Bro, "On the uniqueness of multilinear decomposition of N-way arrays," Journal of Chemometrics, vol. 14, no. 3, pp. 229-239, 2000.

[20] J. B. Kruskal, “Three-way arrays: rank and uniqueness of trilinear decompositions, with application to arithmetic complexity and statistics," Linear Algebra and its Applications, vol. 18, no. 2, pp. 95-138, 1977. 
[21] R. Bro, Multi-way analysis in the food industry: models, algorithms and applications [Ph.D. thesis], University of Amsterdam, Amsterdam, The Netherlands, 1998.

[22] A. Smilde, R. Bro, and P. Geladi, Multi-Way Analysis: Applications in the Chemical Sciences, John Wiley \& Sons, 2004.

[23] P. H. Schönemann, "A generalized solution of the orthogonal Procrustes problem," Psychometrika, vol. 31, no. 1, pp. 1-10, 1966.

[24] G. H. Golub and C. F. Van Loan, Matrix Computations, Johns Hopkins Studies in the Mathematical Sciences, Johns Hopkins University Press, Baltimore, Md, USA, 3rd edition, 1996.

[25] S. Sekizawa, "Estimation of arrival directions using MUSIC algorithm with a planar array," in Proceedings of the IEEE International Conference on Universal Personal Communications (ICUPC '98), pp. 555-559, Florence, Italy, October 1998.

[26] N. Tayem and H. M. Kwon, "L-shape 2-dimensional arrival angle estimation with propagator method," IEEE Transactions on Antennas and Propagation, vol. 53, no. 5, pp. 1622-1630, 2005.

[27] H. Changuel, F. Harabi, and A. Gharsallah, "2-L-shape twodimensional arrival angle estimation with a classical subspace algorithm," in Proceedings of the International Symposium on Industrial Electronics (ISIE '06), pp. 603-607, Montreal, Canada, July 2006.

[28] X. Zhang, X. Gao, and W. Chen, "Improved blind 2D-direction of arrival estimation with L-shaped array using shift invariance property," Journal of Electromagnetic Waves and Applications, vol. 23, no. 5-6, pp. 593-606, 2009. 


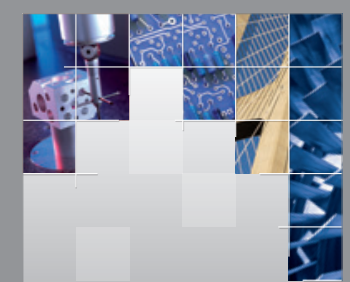

\section{Enfincering}
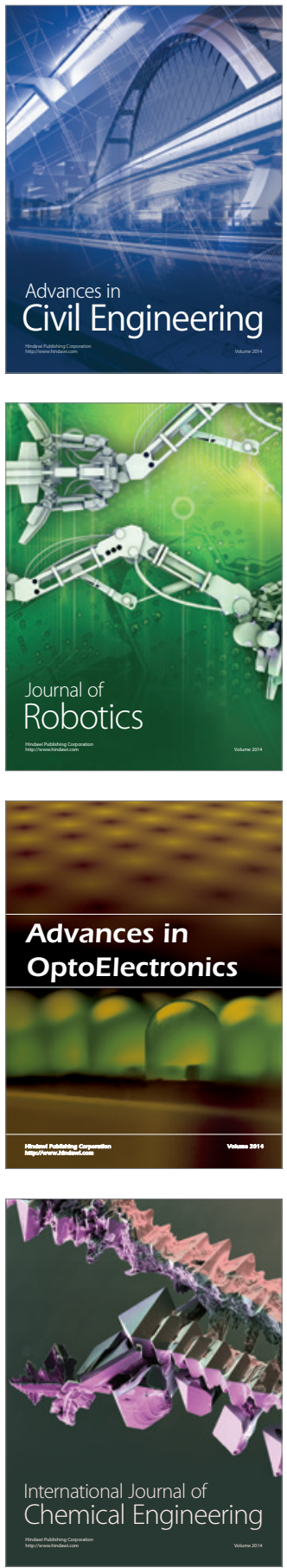

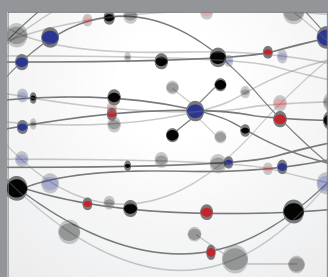

The Scientific World Journal

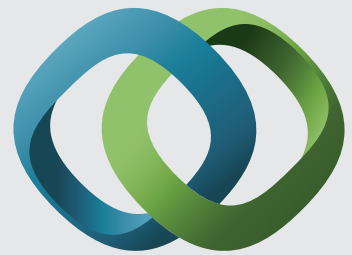

\section{Hindawi}

Submit your manuscripts at

https://www.hindawi.com
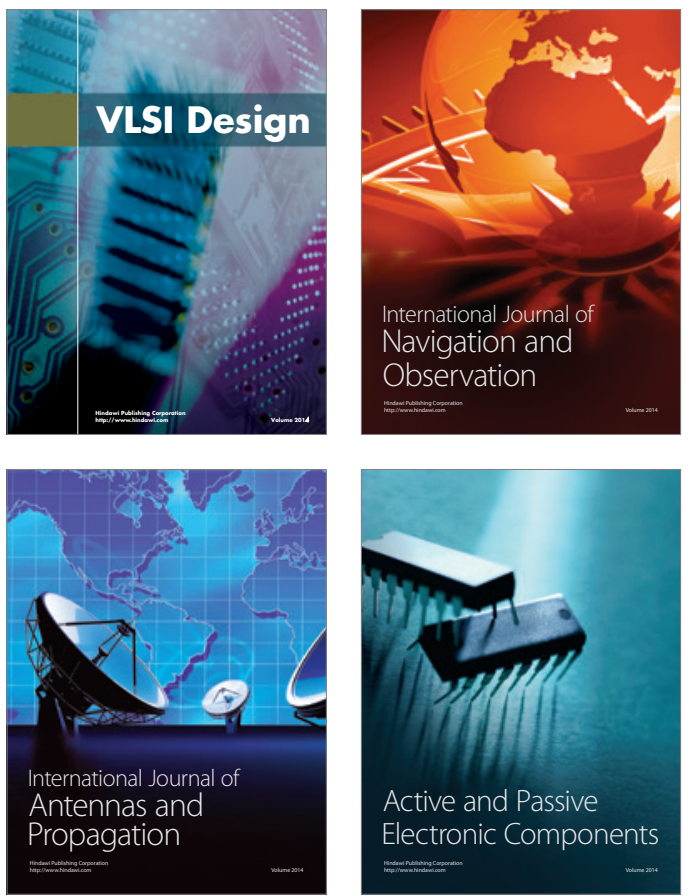
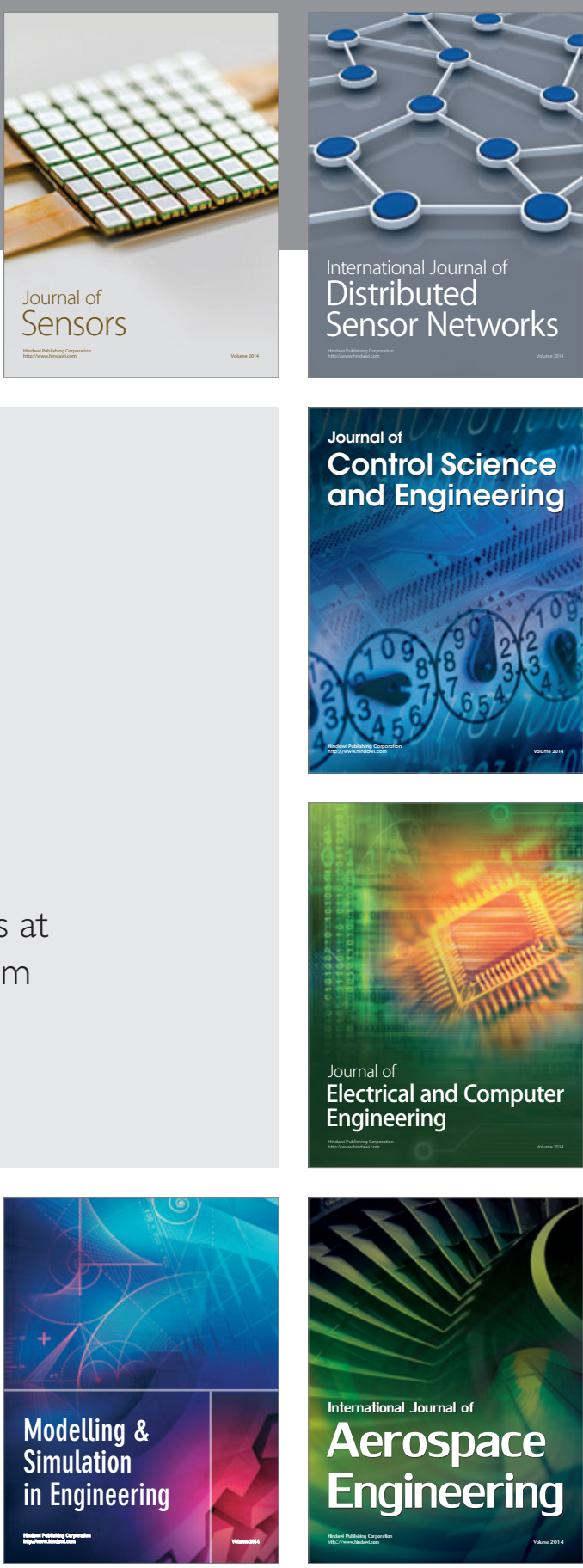

International Journal of

Distributed

Sensor Networks

$-$

Joumal of

Control Science

and Engineering
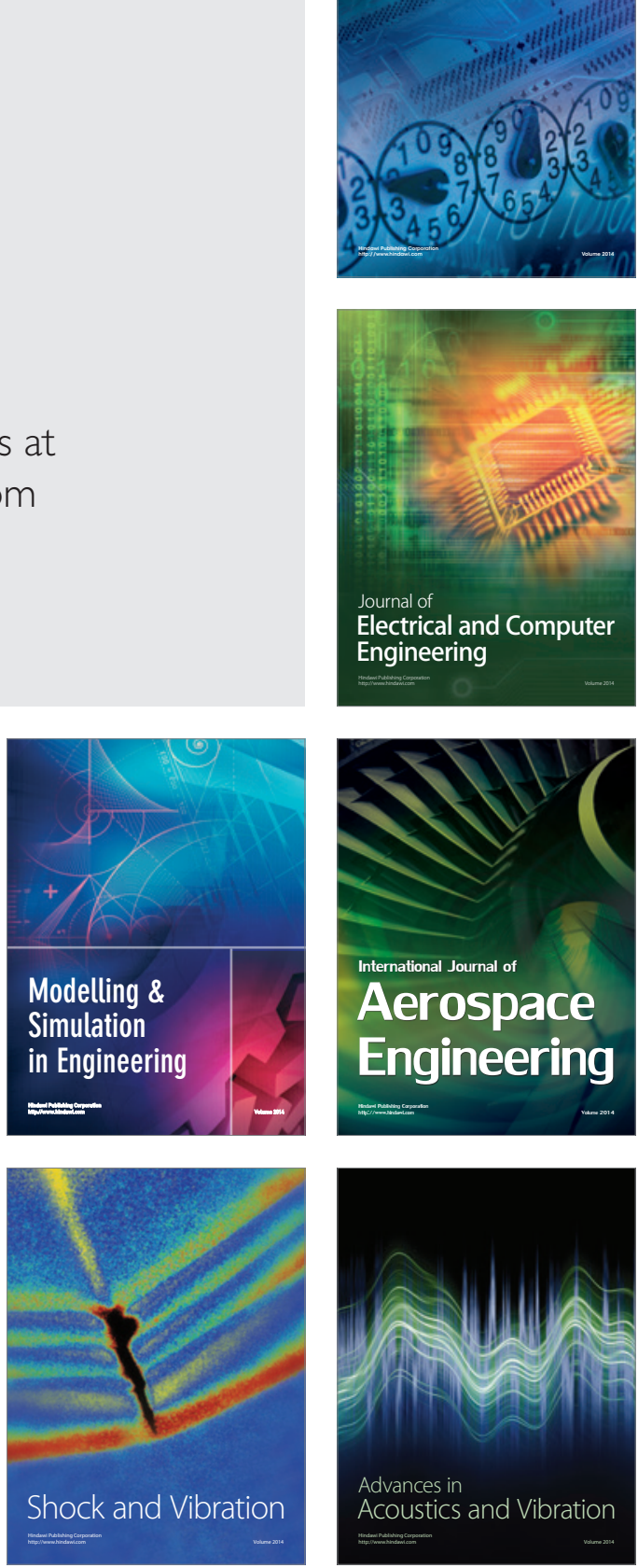